\title{
Algunas relaciones entre la autonomía de la Biología y la emergencia de su Didáctica: consideraciones sobre la complejidad de enseñar una ciencia compleja
}

\author{
Some relationships between Biology's autonomy \\ and the emergence of its didactics: considerations \\ about the complexity of teaching a complex science
}

\author{
Julio Alejandro Castro Moreno ${ }^{1}$ \\ https://orcid.org/0000-0002-5864-0954 \\ Édgar Orlay Valbuena Ussa ${ }^{1}$ \\ https://orcid.org/0000-0003-4185-3862
}

\begin{abstract}
Resumen: En el artículo se plantean algunas relaciones entre la emergencia de la Biología como ciencia autónoma y las primeras propuestas de considerar la Didáctica de la Biología como un campo específico de conocimientos, que, si bien comparte muchas características con la didáctica de las demás ciencias de la naturaleza, igualmente difiere en otros aspectos, los cuales devienen principalmente de las particularidades del estatus epistemológico de la ciencia de lo vivo. En tal sentido, se recurre a algunos autores que han puesto de manifiesto la necesidad de reconocer cuáles serían, entonces, las cualidades que hacen de la Biología una ciencia diferente de, por ejemplo, la Física y la Química. Asimismo, se han retomado las ideas de determinados autores que distinguen la enseñanza de la Biología de la enseñanza de las otras ciencias de la naturaleza.
\end{abstract}

Palabras clave: Enseñanza de la biología. Epistemología. Didáctica de la biología.

\begin{abstract}
In this paper, we discuss some relations between the institutionalization of Biology as an autonomous science and the initial proposals that consider Biology's didactics as a specific field of knowledge. We believe that Biology teaching shares many characteristics with the teaching of other sciences of nature, but those two didactics also differ in several respects. In this way, we claim that those specific features of Biology as science (like epistemological status of science of living things) have important implications in the field of Biology's Didactics. In this regard, we appeal to some authors who have put in mind the necessity to recognize which those would be, and then, consider the qualities that make Biology as a different science to, for example, physics and chemistry. We have also taken up some ideas of certain authors who distinguish between Biology's teaching from teaching of another natural sciences.
\end{abstract}

Keywords: Biology teaching. Epistemology. Didactics of biology.

\footnotetext{
${ }^{1}$ Universidad Pedagógica Nacional (UPN), Departamento de Biología, Bogotá, Colombia.

E-mail: <jcastro@pedagogica.edu.co>.
} 


\section{Introducción}

Este artículo se ha escrito tomando como base una ponencia presentada en el VI Encuentro de Filosofía e Historia de la Ciencia del Cono Sur², la que a su vez se elaboró en el marco del proyecto de investigación "Construcción del Conocimiento Profesional del Profesor de Biología, en El Proyecto Curricular de Licenciatura en Biología de la Universidad Pedagógica Nacional", financiado por el Centro de Investigaciones de la Universidad Pedagógica (CIUP) para la vigencia 2007-2008. Dicho proyecto pretendió indagar las concepciones de los futuros profesores de Biología acerca de su conocimiento profesional, especialmente en lo que atañe al conocimiento biológico y al conocimiento didáctico del contenido biológico. En este sentido, el objetivo central del presente artículo es establecer algunas relaciones entre esos dos ámbitos: el conocimiento que se produce en la Biología y las características distintivas de la didáctica de esa ciencia.

Así las cosas, vale la pena señalar que en un trabajo anterior (CASTRO; VALBUENA, 2007), planteamos el problema de qué conceptos biológicos deberían ser enseñados en la escuela y qué procedimientos serían los más idóneos para tal fin. Una de las conclusiones de dicho trabajo se puede expresar como sigue: el qué y el cómo enseñar deberían estar orientados, en gran medida, pero no exclusivamente, por los discursos históricos y epistemológicos de la Biología. Otra de las conclusiones es que aún está por fundamentarse una disciplina que podamos llamar "Didáctica de la Biología". Consideramos que en este trabajo no avanzamos mucho hacia la configuración de tal disciplina. Sin embargo, creemos dar un paso adelante al reconocer cuáles son las cualidades que le han conferido un estatus epistemológico específico a la Biología, y de qué manera dicho estatus puede orientar la fundamentación de la didáctica de la ciencia de lo vivo.

Del mismo modo, asumimos que las relaciones entre la disciplina científica y la disciplina a ser enseñada son complejas y multidimensionales. Por ello, no tenemos por qué admitir que la enseñanza de una ciencia esté basada únicamente en los aspectos científicos, ya que la didáctica no da cuenta de un simple traslado o "transposición" de saberes de un contexto a otro, sino que se refiere a la transformación e integración de éstos con varios tipos de conocimiento (como el curricular, el práctico, el contextual, el cotidiano, etc.) que hacen posible la construcción del Conocimiento Biológico Escolar. Así las cosas, en este documento retomamos los planteamientos hechos por diversos autores sobre qué hace particular a la Biología como ciencia, para luego hacer algunas reflexiones acerca de cómo aquellas particularidades pueden contribuir a esbozar un discurso teóricamente fundamentado de la Didáctica de la Biología.

\section{La Biología como ciencia autónoma}

En comparación con las demás ciencias de la naturaleza, la institucionalización de la Biología como ciencia autónoma es reciente. En palabras de Coleman (1985), la palabra "biología" se propuso en los primeros años del siglo XIX, de manera independiente por varios naturalistas ${ }^{3}$,

\footnotetext{
${ }^{2}$ Evento realizado en la ciudad de Montevideo, Uruguay, entre el 27 y el 30 de mayo de 2008. 
quienes reconocieron en los "seres organizados" un nuevo problema de conocimiento científico. No obstante, hubo que esperar un siglo para que esta ciencia floreciera. Según autores como Jacob (1999), el proceso de institucionalización de la ciencia de lo vivo se llevó a cabo en el transcurso del siglo en mención, pero tuvieron que transcurrir varias décadas del siglo pasado y muchos eventos trascendentales para que pudiéramos hablar de la Biología como una disciplina con un estatus epistemológico propio. En esta sección mostraremos algunos de los episodios que han transcurrido durante casi dos siglos: desde el bautizo de la Biología hasta el reconocimiento de su autonomía. Veamos brevemente en qué consistieron dichos acontecimientos.

De acuerdo con Foucault (1968), no es posible equiparar la Historia Natural con un estado incipiente de la Biología, es decir que no podemos atribuir una total continuidad entre una y otra, ya que la primera da cuenta de las estructuras visibles, mientras que la segunda se interesa por un objeto de conocimiento hasta entonces (entre los siglos XVIII y XIX) inexistente: la vida, o, en otros términos, la organización propia de los sistemas vivientes (CASTRO, 2012; JACOB, 1999).

Pero también es necesario resaltar uno de los aspectos que posibilitó la emergencia de la Biología, en especial en lo que respecta al distanciamiento de las explicaciones de lo vivo basadas únicamente en los planteamientos de las ciencias fisicoquímicas. Dicho de otra manera, estamos aludiendo al surgimiento de una postura vitalista en contradicción con una visión mecanicista que daba cuenta de los seres vivos. Si bien el vitalismo se convirtió en un obstáculo para explicar el fenómeno viviente en términos que no cayeran en lo metafísico (MAYR, 2006), también es prudente recordar que este compromiso filosófico posibilitó pensar dicho fenómeno como particular e irreductible a las explicaciones de las otras ciencias ya institucionalizadas. En palabras de Jacob (1999, p. 94-95):

El vitalismo funciona como factor de abstracción. La vida juega un papel preciso en el saber. A ella se le interroga en el animal o en la planta, es el objeto mismo del análisis. Representa esa parte desconocida que distingue al organismo de la materia inerte y a la biología de la física. El vitalismo es tan esencial en los inicios de la biología como lo fue antes el mecanicismo; y no sólo para los naturalistas, los fisiólogos o los médicos: también para los químicos que estudian los compuestos orgánicos, es decir, las sustancias que constituyen los seres vivos o que son producidos por ellos.

Sin embargo, es preciso aclarar que entre finales del siglo XIX y principios del XX, la Biología se acercó nuevamente a las ciencias fisicoquímicas, ya que hay varios fenómenos biológicos que no pueden prescindir de lo planteado por esas ciencias. Es el caso, por ejemplo, de la biología molecular y la fisiología. A esta nueva perspectiva se le denominó organicista (MAYR, 1998) y radica en que la Biología no puede reducirse a la física y a la química, pero tampoco puede ignorarlas.

\footnotetext{
${ }^{3}$ Dentro de los que se encuentran Lamarck, en Francia y Treviranus, en Alemania.
} 
Hasta ahora nos hemos referido, exclusivamente, a algunos aspectos históricos que han sido identificados por varios autores como esenciales para hablar del reconocimiento de la Biología como ciencia autónoma. No obstante, hay otros aspectos que debemos traer a colación, ya que tienen que ver con la formalización de un estatus epistemológico propio de la ciencia de lo viviente. Según Mayr (2006), para que la Biología fuera reconocida como una ciencia particular, fue necesario que ocurrieran tres conjuntos de sucesos. El primero de ellos tiene que ver con la refutación de principios erróneos; el segundo hace referencia a la demostración de que algunos postulados de la física no tienen ningún valor explicativo en el mundo viviente; y el tercero está relacionado con la toma de conciencia de la singularidad de los principios básicos de la Biología. Describiremos sintéticamente cada uno de dichos sucesos.

Con respecto al primero, Mayr (2006) resalta la oposición de los biólogos hacia dos ideas erróneas o, al menos, discutibles: el vitalismo y la teleología. Sobre el primero ya nos hemos referido, aunque es necesario agregar que la creencia en una fuerza vital, y el asumir que las sustancias químicas presentes en los organismos son totalmente diferentes de las inorgánicas, o que aquéllas son inexistentes en el mundo inerte, como el protoplasma (MAYR, 1998), no tienen fundamentos científicos, sino que son afirmaciones que se basan en principios filosóficos, más específicamente metafísicos. Sin embargo, coincidimos con Mayr (2006, p. 39) cuando afirma que "Sería ahistórico ridiculizar el vitalismo", teniendo en cuenta lo que expresamos párrafos atrás.

En cuanto a la teleología, el asunto es más complejo, toda vez que existe un fuerte debate (sobre todo epistemológico) al respecto. A ello se suma que, en la enseñanza de la Biología, según López (1996), se utilizan muchas explicaciones de índole teleológica y antropomórfica. Por ahora, y retomando los planteamientos de Mayr (2006, p. 40), podemos decir que "La teleología tiene que ver con la explicación de los procesos que parecen llevar automáticamente a un fin o meta definidos", lo cual puede dar la sensación de que los procesos biológicos están orientados a la consecución de un objetivo predeterminado o, lo que viene a ser su complemento, que las entidades vivientes tienen intencionalidades y conciencia (cualidades que difícilmente se les pueden atribuir a otros organismos diferentes al ser humano).

En ese orden de ideas, Jacob (1999) afirma que el concepto de programa genético, al explicar el desarrollo macroscópico a partir de estructuras y procesos microscópicos, soluciona el problema de la teleología. De igual manera, Monod (1993) propone obviar la finalidad teleológica en contraposición con el concepto de proyecto teleonómico. Para este autor, la teleonomía es una de las propiedades fundamentales de los organismos, puesto que la información "escrita" en el programa genético, se traduce en estructuras y funciones biológicas acordes con las instrucciones codificadas. Por lo tanto, aquello que aparece por azar en el ADN (mutaciones) y que es favorecido por la selección natural, se vuelve necesario para los organismos en los que ocurren dichos cambios. Es decir que los rasgos genotípicos-fenotípicos que tienen éxito en un ambiente dado entran a hacer parte del legado de las próximas generaciones.

En palabras de Monod (1993), los seres vivos son extraños objetos ${ }^{4}$ que están dotados de un proyecto teleonómico, pero inconsciente: dejar descendencia. Valga decir que dicho proyecto es

\footnotetext{
${ }^{4}$ Porque son objetos naturales, como las rocas, pero dan la apariencia de haber sido diseñados (teleológicamente) como los artefactos. 
inherente a los organismos, por lo que podemos afirmar que desde la perspectiva de la teleonomía el objeto y su proyecto son indistinguibles, mientras que la finalidad teleológica es anterior y externa al objeto, lo cual podemos evidenciar en los artefactos creados por la industria humana 5 .

Retomemos ahora los planteamientos de Mayr (2006), específicamente en lo que respecta a las ideas fisicalistas no aplicables a la Biología. Según este autor son cuatro.

La primera de ellas es el pensamiento tipológico o esencialista. Esta idea sostiene que, a diferencia de las ciencias fisicoquímicas, en donde es posible asumir la existencia de elementos idénticos pertenecientes a un conjunto ${ }^{6}$, en Biología no tiene sentido afirmar que todos los individuos pertenecientes a la misma especie poseerían idénticas características y, por ende, serían indistinguibles y reemplazables. En este sentido, Jacob (1998, p. 116-117) afirma que hay una unidad y una similitud en todas las formas de vida, y que dichos aspectos se manifiestan en el nivel molecular. Pero, paradójicamente, aunque todos los seres vivos estamos construidos con los mismos bloques quimicos, éstos se disponen y se organizan de formas muy variadas, aspecto éste que ha dado origen a toda la biodiversidad actual y extinta. Por lo tanto, el pensamiento tipológico no tiene un valor heurístico en la Biología, lo que sí sucede con el pensamiento probabilístico y poblacional que es propio de ésta.

La segunda idea fisicalista no aplicable a la Biología es la del determinismo total, el cual asegura que es posible predecir el estado futuro de un sistema, si se conocen las condiciones actuales del mismo ${ }^{7}$. Según Mayr (2006, p. 44) "La refutación del determinismo estricto y de la posibilidad de la predicción absoluta despejó el campo para el estudio de la variación y de los fenómenos aleatorios, tan importantes en biología".

La tercera de dichas ideas es el reduccionismo ${ }^{8}$, según el cual es factible comprender el funcionamiento de un sistema si se comprenden las propiedades de todas sus partes constituyentes. Cabe hacer notar que Mayr (2006) rechaza este principio, toda vez que una de las cualidades de la Biología es la emergencia de propiedades ausentes en los elementos básicos, pero que se manifiestan a partir de las interacciones entre dichos componentes. Desde este punto de vista, el autor en cuestión critica el uso del reduccionismo en Biología, sin negar que en esta ciencia, en ocasiones, se acuda a una perspectiva analítica, a la hora de hacer determinadas explicaciones.

La cuarta y última idea fisicalista consiste en que las leyes universales hacen parte imprescindible de las explicaciones científicas. A pesar de que algunos autores como Ruse (1979), han defendido la existencia de leyes análogas a las de la física para explicar lo vivo, Mayr (1998, p. 81), insiste que en esta ciencia "los conceptos tienen mucha más importancia que las leyes en

\footnotetext{
${ }^{5}$ Un ejemplo que usa Monod (1993) para ilustrar este punto es con relación a que la idea de cuchillo es anterior a la materialización del instrumento cuchillo. Así, la necesidad de construir el artefacto y la descripción de las propiedades que debería tener, son anteriores a la construcción material del cuchillo. Esto no sucede en el caso de las entidades vivientes.

${ }^{6}$ Aunque debemos decir que no somos tan radicales como Mayr (2006), ya que es claro que en las ciencias físicas juegan un rol preponderante los isótopos. Así, por poner un caso, no hay por qué asumir que todas las moléculas de agua presentes en un recipiente serían idénticas, puesto que ellas están conformadas por los diferentes isótopos del hidrógeno (protio, deuterio y tritio).

${ }^{7}$ En este punto tampoco estamos de acuerdo con Mayr (2206), dado que fue precisamente en la física en donde se postuló el principio de incertidumbre, el cual es totalmente opuesto al determinismo.

${ }^{8}$ Aunque sería mejor hablar de reduccionismos, en plural, siguiendo los planteamientos de Diéguez (2012).
} 
la formación de teorías". Desde nuestra perspectiva, no es fácil asumir la existencia de leyes biológicas, si las entendemos como expresiones universales y matemáticas que enuncian regularidades y predicciones casi infalibles, sobre un fenómeno dado.

Algunos lectores se interrogarán acerca de unas "leyes" que son propias de la Biología: las de Mendel. Pero, ¿son en realidad leyes? Si éstas han de caracterizarse por la regularidad y por tener pocas excepciones, no sería prudente denominar así a los postulados mendelianos, teniendo en cuenta que éstos son aplicables solamente a los organismos sexuales y diploides; a los autosomas y los cromosomas sexuales homólogos (como el par XX en las hembras de los mamíferos); a los genes que poseen solamente dos alelos, los cuales presentan una dominancia completa. Es decir que estas leyes no dan cuenta de fenómenos como la codominancia y dominancia incompleta, sin contar otras situaciones como la expresividad, la penetrancia, la pleiotropía, la epistasis, etc. (VALLEJO, 1998). En pocas palabras, las leyes de Mendel explican la excepción, pero no la regla. Además, lo hacen en términos probabilísticos que escapan a la predicción de las ciencias físicas.

Pasemos ahora al tercer conjunto de hechos que posibilitaron la autonomía de la Biología, es decir al plano de las características propias de esta ciencia. Vale la pena señalar que ya hemos mencionado algunas de ellas, por lo que nos centraremos en un aspecto que consideramos crucial para los propósitos del presente escrito, y que se puede enunciar de la siguiente manera: los sistemas vivientes son complejos. Para Mayr (2006, p. 46), dicha complejidad se expresa en estos términos:

A causa de su complejidad, los sistemas biológicos se encuentran ricamente dotados con capacidades tales como la reproducción, el metabolismo, la replicación, la regulación, la adaptación, el crecimiento y la organización jerárquica. En el mundo inanimado ${ }^{9}$ no existe nada por el estilo.

Igualmente, la complejidad de las entidades vivientes se manifiesta por la gran cantidad de propiedades emergentes que las caracterizan. En tal sentido, cobra relevancia el concepto integrón propuesto por Jacob (1999), que da cuenta de los diversos y sucesivos niveles de integración que ocurren en el seno de la organización de los sistemas vivientes: "La integración es lo que cambia la calidad de las cosas. Una organización suele poseer propiedades que no existen en el nivel inferior. Estas propiedades pueden explicarse por las partes constituyentes, pero no se deducen de ellas" (JACOB, 1999, p. 300).

Según Dawkins (1988), la Biología es el estudio de las cosas complejas, mientras que la física es el estudio de las cosas simples; aunque esto no equivale a decir que esta última sea una ciencia simplista. Este autor hace énfasis en la complejidad de los objetos biológicos, argumentando que si bien un libro de física está lleno de ecuaciones matemáticas (lo cual puede implicar una dificultad para muchas personas), una sola célula del autor de dicho texto es aún

\footnotetext{
${ }^{9}$ Sin embargo, preferimos hablar de mundo inerte, ya que el mundo inanimado nos remite a asumir que también debe haber un mundo animado, y esto nos situaría en un contexto animista. 
más compleja que un hecho explicado en ese material bibliográfico. Así las cosas, los fenómenos de la física son más simples, puesto que se pueden expresar en términos matemáticos, lo que es muy difícil en los procesos biológicos. Para Dawkins, la complejidad biológica se caracteriza, sobre todo, porque no puede haber aparecido solamente por azar, ya que éste es "filtrado" por la selección natural, la que actúa como un relojero ciego.

De acuerdo con Jacob (1988, p. 237), lo complejo de la Biología radicaría en lo siguiente:

El mundo de los seres vivos es el mundo de la complejidad, el resultado de infinitas interacciones de organismos, de células, de moléculas. Para analizar un problema, el biólogo está obligado a concentrar su atención en un fragmento de la realidad, en un pedazo de universo que él aísla arbitrariamente para poder definir una serie de parámetros. En biología, por lo tanto, cualquier estudio empieza por la elección de un «sistema». De esta elección dependen el margen de maniobra del que dispone el experimentador, la naturaleza de las preguntas y la amplitud de los problemas que se puede plantear, y, a menudo, hasta el tipo de respuesta que se puede obtener.

Estas palabras de Jacob (1988) nos sirven para explicitar algunos aspectos del objeto de la Biología. En primer lugar, queremos resaltar que éste no necesariamente debe equipararse a un organismo (uni o pluricelular), sino que puede ser una parte de él (una célula, un organelo, un órgano, etc.), un conjunto de organismos (una población, una comunidad, etc.), una asociación más compleja de organismos (como una colonia de insectos sociales o un liquen) ${ }^{10}$, o una agrupación más amplia que implique el ambiente físico (un ecosistema o la biósfera). En síntesis, aquí la palabra clave es sistema, aunque hemos preferido retomar la nominación de bioorganización sistémica:

[...] la cual da cuenta de diversos sistemas materiales-conceptuales que se organizan en diferentes estados de complejidad (por ejemplo, células y ecosistemas). Por lo tanto, la bioorganización sistémica no se refiere, necesariamente, a organismos-individuos, sino a diversos sistemas abiertos-dinámicos que mantienen su autoorganización, su autorregulación y su autoconstrucción. (CASTRO; VALBUENA; SIERRA, 2009, p. 40).

Por otro lado, el dar cuenta de la complejidad de los sistemas biológicos nos remite al hecho de que para investigarla es necesario acudir a diversos métodos, dentro de los cuales podemos señalar: la experimentación, la observación directa (o mediada por instrumentos), la modelización y la construcción de narraciones históricas. En este orden de ideas, reconocemos

\footnotetext{
${ }^{10}$ En el caso de las colonias de insectos sociales, Hölldobler y Wilson (2014) las han entendido como “superorganismos". Esta idea, sin duda, es una forma de ampliar nuestra comprensión de la complejidad viviente.
} 
una pluralidad disciplinar en la Biología que depende del nivel de bioorganización sistémica que se elija como problema de investigación, y de los métodos que sean más oportunos para llevar a cabo su estudio ${ }^{11}$.

Un último aspecto de la Biología que sólo mencionaremos, pero que reconocemos de gran valor, es el hecho de que esta ciencia, a diferencia de las otras ciencias de la naturaleza, está basada en la historicidad de sus objetos de estudio. En palabras de Wandersee, Fisher y Moody (2000, p. 29), "Los biólogos estudian objetos [...] cuya historia importa". En esa misma vía, Castro (2012) afirma que algo que distingue a la Biología de las demás ciencias naturales es que está basada en la historia, pues todas las entidades vivientes hacen parte de un devenir histórico, en donde la nominación acuñada por Darwin de "descendencia con modificaciones" no tiene parangón en ciencias como la física, la química o la geología. Sin embargo, el hecho de asumir a la evolución como el ejemplo paradigmático de la historicidad de lo vivo, no implica que el proceso evolutivo sea todo lo que importe en la comprensión del mundo viviente (CASTRO, 2013b), pues con ello se estaría yendo en contra de la diversidad ontológica y la pluralidad disciplinar de la Biología, es decir, se estaría negando la complejidad que le es inherente.

Para resumir lo que hemos planteado hasta el momento, vale la pena decir que la cualidad principal de la Biología es que ella da cuenta de la complejidad de los seres vivos, y que dicha complejidad no se puede comprender cabalmente si no se reconoce que ésta ha surgido, se ha mantenido y se ha transformado a través de la historia.

No creemos haber agotado aquí la discusión acerca de las particularidades de la Biología, pero, para efectos de nuestra presentación, solamente diremos que con lo expuesto es suficiente para dejar claro qué hace de la Biología una ciencia autónoma, lo cual, a su vez, tiene implicaciones en la forma en que se asuma su didáctica. Así, llegados a este punto, consideramos que es oportuno relacionar lo expresado hasta ahora con los aspectos que darían cuenta de la Didáctica de la Biología como una disciplina teórica y metodológicamente fundamentada.

\section{Hacia una autonomía de la Didáctica de la Biología}

Hasta aquí nos hemos dedicado a exponer las características que consideramos fundamentales para poder atribuirle un estatus epistemológico propio a la Biología, frente a lo cual asumimos que todas ellas deben ser tenidas en cuenta a la hora de enseñar esta ciencia. Sostenemos que es tarea de los profesores de Biología reflexionar sobre qué particularidades tendrían éstas en el contexto escolar. En este sentido, somos conscientes de que la Biología de los biólogos no es la misma Biología Escolar, aunque pueden tener ciertas similitudes.

En cuanto a las diferencias, es factible anotar que estos dos tipos de conocimiento responden a intereses y finalidades particulares, se construyen en contextos específicos y, además, hay que resaltar que los biólogos se forman tras varios años de estudios universitarios y otros tantos de ejercicio profesional, mientras que los estudiantes de los niveles básico y medio no

\footnotetext{
${ }^{11}$ Dado que no disponemos de espacio para entrar en los detalles de los diferentes métodos de investigación de la biología ( $\mathrm{y}$ en, algunos casos, sus implicaciones en la enseñanza), remitimos al lector interesado a los siguientes trabajos: Castro y Valbuena (2007) y Castro (2013a).
} 
necesariamente van a ser biólogos, por lo que su saber biológico escolar estaría más relacionado con la vida cotidiana, que con el contexto científico propiamente dicho.

Con respecto a las similitudes, coincidimos con Porlán (1999, p. 37) cuando afirma que

[...] algunos principios fundamentales de la producción científica - como la interacción entre teoría y datos empíricos, el trabajo con problemas, el contraste intersubjetivo, la organización interna de los conocimientos, su jerarquización, etc. - son también principios que consideramos consustanciales a la producción de conocimientos en la escuela.

En este momento es importante hacer otros comentarios con respecto a las relaciones entre la Biología y su didáctica. Por un lado, así como la Biología no se institucionalizó como ciencia autónoma hasta bien entrado el siglo XX, del mismo modo nos arriesgamos a afirmar que la didáctica de la Biología aún no ha tomado suficiente distancia de la didáctica de las otras ciencias de la naturaleza. Como sustento de lo anterior, hemos revisado algunos de los trabajos que se han hecho al respecto y hemos concluido, al menos preliminarmente, que aún está por definir cuál sería el estatus epistemológico de la didáctica de la Biología (VALBUENA; CORREA; AMÓRTEGUI, 2012).

En ese sentido, nos ha llamado mucho la atención que algunos libros sobre didáctica de la Biología, que se han publicado en este siglo en el contexto hispanohablante, no digan mayor cosa acerca del tema de qué caracteriza a dicha disciplina ${ }^{12}$. En particular, los trabajos a los que hacemos alusión son: Cañal (2011a, 2011b, 2011c) e Iafrancesco (2005). No obstante, hay que decir que, aunque en otro ámbito, como el anglosajón, tampoco hemos hallado trabajos que expliciten las particularidades de la didáctica de la Biología, sí nos hemos encontrado con la antología coordinada por Kampourakis (2013), en donde se presenta una magnífica colaboración entre filósofos de la Biología y profesores de esta ciencia, en aras de explicitar cómo se puede mejorar la enseñanza la Biología, si ésta se basa en una dimensión filosófica. Este tipo de propuestas conjuntas también se han publicado recientemente en dos números especiales de la revista Science \& Education, dedicados al tema de las relaciones entre filosofía de la Biología y educación en Biología ${ }^{13}$.

A propósito de las revistas especializadas en enseñanza de la Biología ${ }^{14}$, es oportuno decir que éstas son un indicio de que hay una comunidad de especialistas en dicha área, lo cual

\footnotetext{
${ }^{12}$ Esto se podría deber a varias situaciones, como el hecho de que haya un consenso implícito entre la comunidad de profesores de Biología, con respecto a lo que caracteriza la enseñanza de esta ciencia. $\mathrm{O}$, lo que sería lamentable, que dicha comunidad no haya problematizado a fondo el asunto de cuál sería el estatus epistemológico de la didáctica de la Biología. En todo caso, consideramos que esta situación amerita una indagación más exhaustiva que la que hemos planteado en este escrito.

${ }^{13}$ Ver volumen 22, números 1 y 2, 2013.

${ }^{14}$ A nivel mundial destacan muy pocas, pero la mayoría de ellas tiene una amplia trayectoria. Las más reconocidas son: The American Biology Teacher (publicada por The National Association of Biology Teachers, Estados Unidos), Journal of Biological Education (The Royal Society of Biology, Reino Unido), Bio-grafía, escritos sobre la biología y su enseñanza (Universidad Pedagógica Nacional de Colombia), Educación en Biología, (Asociación de Docentes de Ciencias Biológicas de la Argentina) y Revsita da SBEnBio (Associação Brasileira de Ensino de Biologia, Brasil).
} 
se constituye en un aspecto que nos permite afirmar que la didáctica de la Biología es un campo específico de conocimiento o, al menos, que está en proceso de constituirse como tal. Ése es, precisamente, uno de los argumentos de Valbuena, Correa y Amórtegui (2012). Sin embargo, hay que añadir que, de acuerdo con los resultados reportados por estos autores, en los artículos analizados (tomados de diversas revistas especializadas en enseñanza de las ciencias), es posible sostener que la discusión que estamos planteando aún no ha ocupado la atención de los investigadores en la enseñanza de la Biología. En este orden de ideas, vale la pena hacer notar que las investigaciones en el campo citado están más enfocadas en desarrollar estrategias de enseñanza-aprendizaje sobre aspectos puntuales del saber biológico, como la célula, el ecosistema, la genética, la evolución, etc., que a dar cuenta de las especificidades de una didáctica de la Biología.

A pesar de lo anterior, es preciso señalar que, según Valbuena, Correa y Amórtegui (2012, p 88), la diversidad de problemas de investigación evidenciada en los artículos analizados permite plantear "tres grandes agrupaciones (enseñanza-aprendizaje de conceptos específicos, trabajos prácticos y concepciones sobre conceptos biológicos), lo cual podría corresponder a posibles líneas de investigación”. Es decir que esas agrupaciones nos podrían estar mostrando las líneas de investigación que se han constituido, y que posiblemente devienen en la columna vertebral de la didáctica de la Biología, ya se trate de un campo consolidado o en procesos de constitución.

Además de lo dicho hasta ahora, hay que añadir que, por lo menos en el contexto iberoamericano, no hemos hallado la existencia de posgrados específicos sobre enseñanza de la Biología ${ }^{15}$. Por el contrario, en este mismo ámbito hay una gran demanda y oferta de posgrados sobre enseñanza de la física, de la química y de las ciencias de la naturaleza (o ciencias experimentales) en algunos de los cuales se hallan líneas de investigación o "menciones" en enseñanza de la Biología.

Ahora bien, con lo que hemos expresado no queremos sugerir que exista una incompetencia por parte de los profesores de Biología o de las instituciones de educación superior que tienen programas de formación y cualificación de este tipo de profesionales. Muy por el contrario, estamos poniendo de manifiesto que la institucionalización de una disciplina como la didáctica de la Biología se ha visto rezagada con respecto a la didáctica de las otras ciencias de la naturaleza, de manera análoga a como ocurrió con la Biología con relación a las ciencias físicas. En otras palabras, es lógico que el reciente reconocimiento de la Biología como ciencia autónoma haya incidido en la demora del surgimiento de su didáctica. Es posible sustentar esta afirmación con lo planteado por Ogborn et al. (1998, p. 91-92):

Existe un largo camino entre la producción de los conocimientos en la comunidad científica y su aparición definitiva en el aula (o en los periódicos dominicales) [...] El proceso completo lleva una cantidad de tiempo. La teoría sobre los gérmenes que originan las enfermedades ha llegado al conocimiento común más o menos en un siglo; la naturaleza de la herencia

\footnotetext{
${ }^{15}$ Salvo contadas excepciones, como, por citar un caso, la Especialización en Enseñanza de la Biología de la Universidad Pedagógica Nacional de Colombia, programa que entró en funcionamiento en 2007 y dejó de ofertarse en 2013.
} 
a través del ADN llegó a los planes de estudio del bachillerato en el transcurso de una generación; la teoría de la radiación electromagnética no ha llegado aún más allá del nivel universitario a pesar de tener más de un siglo.

Es decir que una vez que se produce el saber en el contexto científico, los maestros, como parte del sistema educativo, contribuirán a decidir qué es lo enseñable de la ciencia, en qué contextos, para qué población, y cómo organizar curricularmente aquello que será enseñado en el ámbito escolar. Pero, según nuestra tesis, esta decisión de los docentes debe estar mediada, inexorablemente, por los discursos históricos y epistemológicos de la disciplina científica a ser enseñada, en este caso la Biología. Con base en esto podemos afirmar que los profesores de ciencias, desde su saber profesional, transformarán didácticamente un conocimiento científico para hacerlo enseñable. Además, dicho saber entrará en diálogo con los saberes propios del estudiante (y otros que confluyen en la escuela) constituyéndose así en un conocimiento escolar.

Ahora consideramos necesario hacer algunas precisiones. Denominamos Conocimiento Biológico (CB) al conjunto de saberes teórico-prácticos elaborados al interior de la comunidad de biólogos. Entendemos por Conocimiento Metadisciplinar de la Biología (CMB) a los discursos históricos, filosóficos, sociológicos, etc., que se producen acerca del CB. Consideramos que el Conocimiento Profesional del Profesor de Biología (CPPB) es el saber particular de los maestros de Biología que los faculta para enseñar esta ciencia, el cual a su vez está compuesto por otros conocimientos, dentro de los que destacamos los dos anteriores y el Conocimiento Didáctico del Contenido Biológico (CDCB), que le permite al profesor hacer enseñable el CB o didactizar este saber. Una de nuestras tesis es que el CMB cumple un rol fundamental para integrar el CB con el CDCB, es decir que éste sirve como puente entre el saber científico y el saber a ser enseñado. Por otra parte, asumimos que el Conocimiento Biológico Escolar (CBE) es el saber de tipo biológico que los estudiantes aprenden en la escuela, aprendizaje que depende de los otros conocimientos descritos, y de la manera en que éstos se integren con otros conocimientos (como el cotidiano) que ya han elaborado los aprendices. Así las cosas, sostenemos que entre el CB y el CBE no se da un simple traslado o transposición de saberes, sino diferentes y complejos procesos de integración y transformación. Es por esto que afirmamos que la Biología de los biólogos no puede ser la misma Biología Escolar. En el Cuadro 1 se sintetiza lo planteado en este párrafo.

Desde esta perspectiva, el profesor de Biología sería un mediador entre, al menos, tres ámbitos (científico, cotidiano y escolar), para lo cual es necesario que recurra a la historia y la epistemología de esta ciencia, toda vez que "Conocer las respuestas distintas -o incluso contradictorias - que se han dado [a lo largo de la historia de la Biología] y los obstáculos que han debido superarse para construir determinadas teorías o conceptos, puede resultar útil para entender las dificultades que tienen los estudiantes" (JIMÉNEZ, 2003, p. 122). Así pues, es imprescindible que empecemos a esbozar las características principales de una didáctica de la Biología que posibilite una construcción compleja del CBE.

En lo que respecta su estatus epistemológico, es necesario entrar en diálogo con lo que expresamos en el apartado anterior. Por ejemplo, debemos empezar a reflexionar acerca de las dificultades de enseñanza-aprendizaje que implican los diferentes niveles de complejidad inherentes al objeto biológico (la bioorganización sistémica), y los diversos tipos de procederes que son pertinentes para lograr su comprensión en el aula. Si lo anterior nos conduce a 
reconocer que la Biología tiene particularidades que la diferencian de las otras ciencias de la naturaleza, entonces esto conlleva el reconocimiento de que esta ciencia amerita una didáctica que dé cuenta de dichas especificidades.

Cuadro 1. Diferentes tipos de conocimiento implicados en la enseñanza de la Biología

\begin{tabular}{|l|l|}
\hline \multicolumn{1}{|c|}{ Tipo de conocimiento } & \multicolumn{1}{c|}{ Características } \\
\hline Conocimiento Biológico (CB) & $\begin{array}{l}\text { Conocimiento producido al interior de la comunidad de } \\
\text { biólogos }\end{array}$ \\
\hline $\begin{array}{l}\text { Conocimiento Metadisciplinar de la } \\
\text { Biología (CMB) }\end{array}$ & $\begin{array}{l}\text { Conocimiento sobre el conocimiento biológico } \\
\text { (metaconocimiento), producido principalmente en la filosofía, } \\
\text { la historia y la sociología de la biología }\end{array}$ \\
\hline $\begin{array}{l}\text { Conocimiento Profesional del } \\
\text { Profesor de Biología (CPPB) }\end{array}$ & $\begin{array}{l}\text { Conocimiento propio del quehacer profesional del profesor de } \\
\text { biología, que lo faculta para hacer enseñable el saber biológico } \\
\text { en diferentes niveles educativos. }\end{array}$ \\
\hline $\begin{array}{l}\text { Conocimiento Didáctico del } \\
\text { Contenido Biológico (CDCB) }\end{array}$ & $\begin{array}{l}\text { Es un componente fundamental del CPPB, el cual consiste } \\
\text { en el conocimiento que le permite al profesor transformar } \\
\text { contenidos particulares de la Biología en objeto de enseñanza. }\end{array}$ \\
\hline $\begin{array}{l}\text { Conocimiento Biológico Escolar } \\
\text { (CBE) }\end{array}$ & $\begin{array}{l}\text { Es el conocimiento relacionado con la biología que se } \\
\text { construye, por parte de los estudiantes, en el contexto escolar. }\end{array}$ \\
\hline
\end{tabular}

Fuente: elaboración de los autores.

En consecuencia, consideramos que el proceso de selección y transformación del CB que el maestro hace para facilitar la construcción de un CBE por parte de sus estudiantes, se verá altamente beneficiado si el profesor se preocupa por comprender aspectos centrales de la filosofía y la historia de la disciplina a enseñar (o sea, el CMB). Pero lo anterior, aunque necesario, no es suficiente, ya que también debe conocer, entre otras cosas, las concepciones, intereses, motivaciones y preocupaciones que sus estudiantes tienen con respecto al conocimiento de lo vivo.

En síntesis, afirmamos que el conocimiento particular de la didáctica de la Biología es el CDCB (VALBUENA, 2007), lo cual implica que esta disciplina emergente produce conceptos específicos que son los que le dan competencia profesional al profesor para hacer "enseñable" la Biología. O sea, que este conocimiento le permite al profesor reflexionar sobre su práctica docente, en aras de que sus estudiantes construyan un CBE acorde con las finalidades de enseñanza. Lo anterior conlleva, entre otros, los siguientes aspectos:

- la indagación, análisis didáctico y utilización de las concepciones de los estudiantes acerca de la ciencia, la Biología y los diferentes conceptos biológicos;

- la identificación y organización de conceptos estructurantes específicamente biológicos, como los definidos en Castro y Valbuena (2007);

- la organización de los diferentes tipos de contenidos de enseñanza (conceptuales, actitudinales y procedimentales);

- la identificación y superación de problemas particulares (obstáculos epistemológicos) de la enseñanza de la Biología, tales como el mecanicismo, el reduccionismo, el antropocentrismo, 
la no relación entre los procesos biológicos evidenciables en el mesocosmos con los procesos a nivel microscópico, las explicaciones teleológicas y antropomórficas, etc.;

- la identificación de finalidades de la enseñanza de la Biología;

- las estrategias propias de la enseñanza de la Biología, como las salidas de campo, el trabajo práctico en el laboratorio (con las implicaciones de la experimentación particular con organismos), la modelización de fenómenos biológicos, la construcción de narraciones históricas, etc. ${ }^{16}$;

- el reconocimiento de las relaciones Ciencia-Tecnología-Sociedad-Ambiente (CTSA), dadas las características integradoras y de aplicación de los conceptos biológicos.

De esta manera, reiteramos que un aspecto esencial del CPPB es el conjunto de saberes, prácticas, procedimientos, presupuestos, conceptos, finalidades, formas de explicar, principios, teorías, etc., propios de la Biología como ciencia autónoma, es decir, el CB. Pero no basta con que el profesor tenga un dominio de estos ámbitos, también es fundamental que comprenda aspectos didácticos de la Biología, que hemos denominado como CDCB, dentro de los cuales podemos destacar: qué es lo enseñable de esta ciencia, con qué finalidad se enseñan unos contenidos específicos, en qué contexto, para qué población, bajo qué intereses, orientado por qué problemáticas, de qué manera se deben organizar curricularmente dichos contenidos, cuáles son las estrategias más idóneas para evaluar los aprendizajes, etc.

A nuestro modo de ver, una interesante manera de interrelacionar estos dos componentes del CPPB es a través de la reflexión sobre los ámbitos histórico y filosófico de esta ciencia, es decir a través del CMB. Reconocer qué hace particular a la Biología y qué dificultades (como los obstáculos epistemológicos) han tenido que ser superadas para que esta ciencia sea considerada como autónoma, puede ayudarnos a reflexionar acerca de qué enseñar de la Biología y cómo hacerlo. Asumir la construcción del CPPB en estos términos, es una condición sine qua non para lograr que nuestros estudiantes construyan un CBE menos informativo y más complejo.

\section{A manera de conclusión}

Hemos puesto de manifiesto que algunos biólogos, interesados en los aspectos histórico-epistemológicos de la Biología, han conceptualizado cuáles son las particularidades de ésta como ciencia, aspectos que le han conferido autonomía. En ese sentido, se ha hecho evidente un distanciamiento con las otras ciencias de la naturaleza, entre otras cosas, porque sus objetos de conocimiento difieren de la bioorganización sistémica. Si se nos pidiera definir, en una palabra, cuál es la principal característica del objeto biológico, diríamos: complejidad. Tal propiedad la explicitamos ampliamente párrafos atrás.

\footnotetext{
${ }^{16}$ En lo que atañe a una estrategia didáctica para la enseñanza-aprendizaje de la Biología a través de la investigación del entorno natural, en donde se llevaron a cabo algunos de los procedimientos señalados, véase Castro (2005).
} 
Dicho lo anterior, si se nos preguntara cuál sería el objeto de la Didáctica de la Biología, diríamos: la complejidad de enseñar cuestiones biológicas. Es decir que el quehacer de esta disciplina ha de centrarse en dar cuenta de la complejidad que implica enseñar la complejidad del mundo viviente. Como lo plantean Wandersee, Fisher y Moody (2000, p. 30, traducción nuestra), "La amplitud y la complejidad de la biología, la interconexión de los conocimientos en muchos niveles diferentes, y la naturaleza invisible de muchos procesos clave de la biología, hacen de ésta un tema particularmente difícil de enseñar y de aprender".

Ya los historiadores y epistemólogos de la Biología nos han abierto una vía por la cual empezar a comprender en qué radica la autonomía de esta ciencia. Nuestra tarea, ahora, es empezar a cimentar las bases de una Didáctica Autónoma de la Biología y fortalecer las líneas de investigación que de ella han surgido. Entendemos que no es una tarea fácil, y que no basta con las reflexiones histórico-epistemológicas y didácticas hechas aquí. Dicha disciplina, como hemos visto, entraña una complejidad doble, por lo que es imperativo duplicar los esfuerzos. Emprender la tarea de conferir autonomía ${ }^{17}$ a la didáctica de ciencia de lo vivo, no puede ser un proceso idéntico al que implicó el reconocimiento de la autonomía de la Biología con respecto a las otras ciencias de la naturaleza. No obstante, tenemos mucho que aprender de ese "movimiento independentista". No echemos en saco roto dos siglos de historia.

\section{Referencias}

CAÑAL, P. (Coord.). Biología y geología: complementos de formación disciplinar. Barcelona: Graó, 2011a.

CAÑAL, P. (Coord.). Biología y geología: investigación, innovación y buenas prácticas. Barcelona: Graó, 2011c.

CAÑAL, P. (Coord.). Didáctica de la biología y la geología. Barcelona: Graó, 2011b.

CASTRO, J. A. La biología como ciencia histórica: el caso de la evolución biológica.

Bio-Grafía: escritos sobre la biología y su enseñanza, Bogotá, v. 5, n. 9, p. 19-37, 2012.

Disponible en: < http://dx.doi.org/10.17227/20271034.vol.5num.9bio-grafia19.37>.

Visitado el: 19 jul. 2016.

CASTRO, J. A. Conocimiento práctico, historia, filosofía y enseñanza de la biología: el caso de la herencia biológica. Tecné, Episteme y Didaxis, Bogotá, n. 34, p. 103-125, 2013 a.

CASTRO, J. A. La investigación del entorno natural: una estrategia didáctica para la enseñanza-aprendizaje de las ciencias naturales. Bogotá: Universidad Pedagógica Nacional; Fundación Francisca Radke, 2005.

\footnotetext{
${ }^{17}$ Hay que aclarar que cuando hacemos un llamado a la autonomía, ello no significa una separación total y una incomunicación con respecto a la didáctica de ciencias como la física y la química. ¡Insistimos en la necesidad de reconocer las particularidades de la Biología, las cuales demandan una didáctica propia! 
Algunas relaciones entre la autonomía de la Biología ...

CASTRO, J. A. ¿Nada en biología tiene sentido si no es a la luz de la evolución? Ciência \& Educação, Bauru, v. 19, n. 4, p. 971-994, 2013b.

CASTRO, J. A.; VALBUENA, E. ¿Qué biología enseñar y cómo hacerlo?: hacia una resignificación de la biología escolar. Tecné, Episteme y Didaxis, Bogotá, n. 22, p. 126-145, 2007.

CASTRO J. A.; VALBUENA, E.; SIERRA, C. A. La hipótesis de progresión como enfoque para el estudio de las representaciones sobre la biología. In: VIALES, R.; AMADOR, J.; SOLANO, F. (Comp.). Concepciones y representaciones de la naturaleza y la ciencia en América Latina. San José: SIEDIN; Universidad de Costa Rica, 2009. p. 35-42.

COLEMAN, W. La biología en el siglo XIX: problemas de forma, función y transformación. México: Fondo de Cultura Económica, 1985.

DAWKINS, R. E1 relojero ciego. Barcelona: Labor, 1988.

DIÉGUEZ, A. La vida bajo escrutinio: una introducción a la filosofía de la biología. Barcelona: Biblioteca Buridán, 2012.

FOUCAULT, M. Las palabras y las cosas. México: Siglo XXI, 1968.

HÖLLDOBLER, B.; WILSON, E. O. E1 superorganismo: belleza y elegancia de las asombrosas sociedades de insectos. Buenos Aires: Katz, 2014.

IAFRANCESCO, G. Didáctica de la biología: aportes a su desarrollo. Bogotá: Magisterio, 2005.

JACOB, F. La estatua interior. Barcelona: Tusquets, 1988.

JACOB, F. El ratón, la mosca y el hombre. Barcelona: Crítica, 1998.

JACOB, F. La lógica de lo viviente: una historia de la herencia. Barcelona: Tusquets, 1999.

JIMÉNEZ, M. P. La enseñanza y el aprendizaje de la biología. In: JIMÉNEZ, M. P. (Coord.). Enseñar ciencias. Barcelona: Graó, 2003. p. 121-146.

KAMPOURAKIS, K. (Ed.). The philosophy of biology: a companion for educators.

Dordrecht: Springer, 2013.

LÓPEZ, A. La explicación teleológica en la enseñanza y aprendizaje de la biología. In:

CARRETERO, M., et al. Construir y enseñar las ciencias experimentales. Buenos Aires: Aique, 1996. p. 153-172.

MAYR, E. Así es la biología. Madrid: Debate, 1998.

MAYR, E. Por qué es única la biología: consideraciones sobre la autonomía de una disciplina científica. Buenos Aires: Katz, 2006.

MONOD, J. El azar y la necesidad: ensayo sobre la filosofía natural de la biología moderna. Barcelona: Tusquets, 1993.

OGBORN, J. et al. Formas de explicar: la enseñanza de las ciencias en secundaria. Madrid: Santillana, 1998. 
PORLÁN, R. Hacia un modelo de enseñanza-aprendizaje por investigación. In: KAUFMAN, M.; FUMAGALLI, L. Enseñar ciencias naturales: reflexiones y propuestas didácticas. Buenos Aires: Paidós, 1999. p. 23-64.

RUSE, M. La filosofía de la biología. Madrid: Alianza, 1979.

VALBUENA, E. E1 conocimiento didáctico del contenido biológico: estudio de las concepciones disciplinares y didácticas de futuros docentes de la Universidad Pedagógica Nacional, Colombia. 2007. Tesis (Doctorado en Didáctica de las Ciencias Experimentales) Universidad Complutense de Madrid, Madrid, 2007.

VALBUENA, E.; CORREA, M.; AMÓRTEGUI, E. La enseñanza de la biología ¿un campo de conocimiento?: estado del arte 2007-2008. Tecné, Episteme y Didaxis, Bogotá, n. 31, p. 67-9, 2012.

VALLEJO, F. La tautología darwinista y otros ensayos de biología. Madrid: Taurus, 1998.

WANDERSEE, J.; FISHER, K.; MOODY, D. The nature of biology knowledge. In: FISCHER, K.; WANDERSEE, J.; MOODY, D. Mapping biology knowledge. Dordrecht: Kluwer, 2000. p. 25-37.

Artigo recibido en 18/02/2017. Aceptado en 24/10/2017. 